\title{
Erörterungen zu den Handlungsempfehlungen
}

Das Projekt HUMAN wurde in dem Bestreben umgesetzt, Empfehlungen zum fachlichen Umgang mit mediatisierter sexualisierter Gewalt in Kindheit und Jugend $\mathrm{zu}$ entwickeln. Um dabei dem Anspruch gerecht $\mathrm{zu}$ werden, auf die Bedürfnisse von Fachkräften und Betroffenen einzugehen, haben Gewaltbetroffene und deren Angehörige, Fachkräfte der spezialisierten Fachberatung sowie interdisziplinäre Expert*innen aus Wissenschaft und Praxis an deren Entwicklung partizipiert. ${ }^{1}$ Als Stellvertreter*innen der adressierten Zielgruppe(n) haben sie ihre (Erfahrungs-)Expertise in unterschiedlicher Form eingebracht, um auf diese Weise Sorge für die Praxisnähe zu tragen. Nicht zuletzt deshalb verstehen sich die Handlungsempfehlungen als Ansätze. Als solche müssen sie auf die eigene Arbeit, genauer den Einzelfall, übertragen werden. Durch veranschaulichende Fallbeispiele angeregt, stehen die persönliche Auseinandersetzung mit und die Reflexion von fachlichen Fragen und Herausforderungen im Vordergrund. Es geht also darum, die Weichen zu stellen, um die professionelle Haltung weiterzuentwickeln, professionelles Handeln situativ abzuwägen, zu begründen und in herausfordernden Situationen Handlungssicherheit zu erlangen (Kunz et al., 2016). Das setzt allerdings zweierlei voraus; zum einen die Beschäftigung mit eigenen Gefühlen, Vorannahmen, Assoziationen, Interpretations- und Deutungsmustern.

\begin{abstract}
„Wir sind schnell in der Gefahr als Profis, da etwas hineinzudeuten oder eigene Fantasien auch zu entwickeln, was könnte das sein, ist dahinter eine schwerwiegendere Problematik, ist da was mit Manipulation, ist da was mit einer sexuellen Devianz [...] oder was auch immer? Und ich glaube, zu diesem professionellen Handeln gehört
\end{abstract}

\footnotetext{
${ }^{1}$ Partizipation sei in Orientierung an Bergold und Thomas (2012) sowie Bahls et al. (2018) als die Beteiligung der adressierten Zielgruppen an forscherischen Prozessen wie der Erhebung des Datenmaterials und der Darstellung der Ergebnisse verstanden.
} 
eben auch, das erst mal in den Griff zu kriegen, also die eigenen Fantasien, die eigenen Vorstellungen, wo auch immer die herkommen, aus der eigenen Biografie, aus der eigenen Sozialisation, ja, Moralentwicklung und der eigenen Ethik, die ja bei uns auch mit der Professionalität ganz stark einhergeht."

Rechtspsychologe, Gutachter, FGI

Zum anderen braucht es ein Verständnis für die Aufträge, Rechte und Pflichten aller in den Unterstützungs- und Hilfeprozess eingebundenen Akteur*innen (z. B. Schule, Jugendamt, Polizei, Beratungsstelle). Schließlich resultieren hieraus Betrachtungs- und Herangehensweisen, die sowohl die eigene Arbeit als auch die Zusammenarbeit prägen. Mit den Fallbeispielen versuchen wir, entsprechende Reflexionsräume zu öffnen. Das bedeutet gleichzeitig aber auch, dass die Handlungsempfehlungen weder allgemeingültige Handlungsanweisungen, Handlungsleitfäden oder Checklisten darstellen noch den Anspruch eines politischen Positionspapiers erfüllen. Sie zeigen stattdessen Möglichkeiten auf, um innerhalb der gegebenen strukturellen und rechtlichen Rahmenbedingungen der Kinderund Jugendhilfe von mediatisierter sexualisierter Gewalt betroffenen Kindern und Jugendlichen bestmöglich zu helfen.

\subsection{Wichtige Lektürehinweise}

Den Handlungsempfehlungen geht stets eine Diskussion des Fallbeispiels voraus. Sie dient der Erörterung und Reflexion fachlicher (offener) Fragen und als herausfordernd erlebter Momente. Wir empfehlen den Lesenden, das jeweilige Fallbeispiel zunächst auf sich wirken zu lassen und sich anschließend Zeit und Raum für die angeführten Reflexionsfragen zu nehmen. Die Lektüre kann aus unterschiedlichen Gründen Gefühle und Widerstände auslösen. Ein Grund hierfür können entweder fehlende oder erst in der Fortsetzung des Fallbeispiels bereitgestellte Informationen sein, wodurch die fachliche Einordnung und Überlegungen zu adäquater Hilfe erschwert werden. Dahinter steht nicht die Absicht, die Lesenden zu verärgern, sondern eine Reflexion persönlicher Vorannahmen, Assoziationen und Interpretationsansätze anzustoßen.

Die Fälle stehen jeweils exemplarisch für eine typische und wiederkehrende Situation im Umgang mit mediatisierter sexualisierter Gewalt. Die Handlungsempfehlungen sind in ihrem Kern generalisierbar. Sie sind jedoch insoweit auf den zugrunde liegenden Fall begrenzt, als bestimmte Fallmerkmale spezifische Intervention(en) fordern. Unter Berücksichtigung all dessen setzt das Anwenden 
und Umsetzen der Handlungsempfehlungen einen Übertrag auf andere Fallkonstellationen voraus. Nicht zuletzt aus diesem Grund haben wir uns dazu entschieden, einige Fallbeispiele zu splitten und die Fortsetzung des Fallbeispiels erst im Kapitelverlauf einzuführen. Mit der Fortsetzung kann sich die fachliche Bewertung der Gewalt und der Interventionen verändern. Das ist insoweit beabsichtigt, als der erste Teil bereits eine in sich geschlossene „Gewaltgeschichte" darstellt. Mit der Fortsetzung gewinnt der Fall an Komplexität. Zur besseren Nachvollziehbarkeit sei dies anhand eines Beispiels erläutert: In Teil 1 des Fallbeispiels wird darüber berichtet, dass ein Penisbild des jugendlichen Adressaten im Fußballverein kursiert. Mit der Fortsetzung des Fallbeispiels erfahren wir, dass das Penisbild im Zusammenhang mit dem zwei Jahre zurückliegenden sexuellen Missbrauch des Jungen entstand. Die Gewaltwiderfahrnisse sind nun anders einzuordnen, womit andere Maßnahmen zur Intervention geboten sind. Durch das Splitten des Fallbeispiels sind die Handlungsempfehlungen insoweit umfassender, als sie für beide Fallkonstellationen ausgesprochen werden. Gleichzeitig steigt der Informationsgehalt und damit die Eindeutigkeit von Fallfakten.

Des Weiteren baut jede Falldiskussion auf die vorherige auf, indem an definierte Fachbegriffe, vermitteltes Wissen, skizzierte Diskurse und ausgesprochene Empfehlungen angeknüpft wird. Die Falldiskussionen der Kap. 4 bis 10 sind zwar nichtsdestotrotz unabhängig voneinander lesbar, doch womöglich ist die Lektüre - abhängig von persönlichem Vorwissen - anspruchsvoll.

Um Informationen und Argumentationen zu veranschaulichen und zu belegen, zitieren wir wie nachfolgend skizziert aus den folgenden Datenquellen, die zu Projektzwecken erhoben bzw. entwickelt wurden:

- Falldokumentationen (Falldokumentation, Kapitel- und Fallnummer; Beispiel: Falldokumentation 5.1)

- Interviews (Interview, Angabe zur Interviewperson und ihres Geschlechts; Beispiel: Interview, Mutter einer Gewaltbetroffenen)

- Focus Group Interviews (Fachdisziplin und Tätigkeitsgebiet des*der zitierten Expert*in, FGI; Beispiel: Soziologe, spezialisierte Fachberatung, FGI)

\subsection{Entstehung der Handlungsempfehlungen}

Der Arbeit an den Handlungsempfehlungen ging das Entwickeln der ihnen zugrunde liegenden Fallbeispiele voraus. Um diese möglichst praxisnah und für 
mediatisierte sexualisierte Gewalt charakteristisch auszuarbeiten, bedurfte es auf unserer Seite einer umfassenden Einarbeitung.

\subsubsection{Identifizieren wiederkehrender fachlicher Fragen}

$\mathrm{Zu}$ diesem Zweck kooperierten wir mit zehn Fachberatungs- und Präventionsstellen, die für eine Aufwandsentschädigung und im Einverständnis der Gewaltbetroffenen sowie deren Erziehungsberechtigter insgesamt 46 pseudonymisierte $^{2}$ Fälle mediatisierter sexualisierter Gewalt gegen Kinder und Jugendliche dokumentierten. In ihrem Aufbau folgten die Falldokumentationen einer vorgegebenen Struktur, die Beschreibungen a) der sexualisierten Gewalthandlungen, b) der Funktion/Bedeutung digitaler Medien, c) der Betroffenen, Gewaltausübenden, sozialen Bezugssysteme, d) des sozialen Kontexts, e) zur Aufdeckung, f) der unmittelbaren Reaktionen nach der Aufdeckung, g) der Belastungen und Befindlichkeit der Betroffenen sowie h) der geleisteten Hilfe und (pädagogischen) Interventionen umfassten (Kärgel \& Vobbe, 2020).

Bei der Arbeit mit den Falldokumentationen fokussierten wir Momente, die von den Fachkräften im Rahmen der Fallbearbeitung wiederholt als herausfordernd erlebt wurden. In der Sozialen Arbeit werden solcherlei als ,typisch“ empfundenen und „,im professionellen Geschehen wiederkehrende Situationen“ als Schlüsselsituationen bezeichnet. ,Sie zeichnen sich einerseits durch generalisierbare und verallgemeinerbare Merkmale aus, die für eine gelingende Professionalität als bedeutsam erachtet werden, andererseits werden die erlebten Situationen in ihrer spezifischen Ausprägung beschrieben." (Tov et al., 2016, S. 40). Schlüsselsituationen mediatisierter sexualisierter Gewalt sind in unserem Projektzusammenhang demgemäß Situationen, in welchen sich Fachkräfte regelmäßig wiederfinden. Die Situationsmerkmale sind dabei teils generalisierbar und verallgemeinerbar (z. B. hinsichtlich der Bedeutung und Funktion digitaler Medien, der Folgewirkungen und Belastungen), teils fallspezifisch (z. B. Gewaltkontext, Alter und Geschlecht der Gewaltbetroffenen). Sämtliche Schlüsselsituationen kennzeichnen sich dadurch, dass sie Ambivalenzen und Unsicherheiten, Dilemmata und Spannungsfelder oder Herausforderungen und Überforderungen abbilden.

\footnotetext{
${ }^{2}$ Die Falldokumentationen wurden unter Einhaltung der Datenschutzgrundverordnung pseudonymisiert übermittelt. Die Verantwortung der Pseudonymisierung oblag zwecks des Schutzes der Betroffenen den Fachstellen. Eine Verfahrensbeschreibung zur Pseudonymisierung wurde durch das Projektteam bereitgestellt. Sie beruht auf den Empfehlungen der durch das Ministerium für Wissenschaft, Forschung und Kunst des Landes Baden-Württemberg geförderten Informationsplattform forschungsdaten.info (2019).
} 
Dabei wurden die Wahrnehmungen der Falldokumentierenden ebenso wie der Gewaltbetroffenen, deren Angehöriger, weiterer beteiligter Personen und/oder Institutionen und Forschenden berücksichtigt. Durch das Zusammenführen dieser Perspektiven arbeiteten wir unterschiedliche Ansichten auf Bedeutungen, Interpretationen und Lösungswege heraus, die sich aus Perspektive der Fachkräfte in der alltäglichen Fallbearbeitung als konfliktbehaftet erweisen.

\subsubsection{Entwickeln idealtypischer Fallvignetten}

Diese konfliktbehafteten Schlüsselsituationen mediatisierter sexualisierter Gewalt wurden anschließend in eine idealtypische Fallgeschichte (Fallvignette) eingebettet, indem wir beispielsweise den Gewaltbetroffenen und Gewaltausübenden Zuschreibungsmerkmale (z. B. Alter, Geschlechtlichkeit) zugewiesen sowie Intervention(en) und Reaktionen des sozialen Umfelds skizziert haben. Hierbei haben wir uns stets an der empirischen Verteilung orientiert. Somit handelt sich bei den Fallvignetten um reine Rekonstruktionen, die auf Basis des Datenmaterials als „Idealtyp“ (Kelle \& Kluge, 2010) entwickelt wurden. Dementsprechend wurde aus mehreren Falldokumentationen, die eine Schlüsselsituation bestmöglich repräsentieren, jeweils eine modellhafte Fallvignette konstruiert. ${ }^{3}$ Rückschlüsse auf originale Fälle sind somit nicht möglich. Die Fallvignetten orientieren sich dennoch an der Problematik, Darstellungsform und dem sprachlichen Duktus des Datenmaterials, ohne jedoch dem Wortlaut eines einzelnen Originalfalls gesamtzusammenhängend $\mathrm{zu}$ entsprechen. Anhand der beiden folgenden Beispiele in Tab. 3.1 sei veranschaulicht, in welcher Weise originäre Fallmerkmale in die rekonstruierten Schlüsselsituationen bzw. Fallvignetten eingearbeitet wurden.

\subsubsection{Identifizieren von Handlungsansätzen}

$\mathrm{Zu}$ den Fallvignetten sind wir im Rahmen von acht Focus Group Interviews ${ }^{4}$ (FGI) mit 22 interdisziplinären Expert*innen aus Wissenschaft und Praxis in

\footnotetext{
${ }^{3}$ Eine unvollständige Berücksichtigung potenzieller Ausprägungen der Kontextvariablen stellt daher kein Versäumnis des Projektteams dar. Sie begründet sich vielmehr im Datenmaterial.

${ }^{4}$ Ein Focus Group Interview ist ein moderiertes Diskussionsverfahren, bei dem eine Kleingruppe durch einen Informationsinput zur Diskussion über ein bestimmtes Thema angeregt wird (Bohnsack \& Przyborski 2009).
} 
Tab.3.1 Beispiele zur Veranschaulichung der idealtypischen Rekonstruktionen

\begin{tabular}{|c|c|}
\hline $\begin{array}{l}\text { Zitat aus einer originären } \\
\text { Falldokumentation }\end{array}$ & $\begin{array}{l}\text { Auszug aus einer idealtypischen } \\
\text { Schlüsselsituation }\end{array}$ \\
\hline $\begin{array}{l}\text { „Alle Voraussetzungen für das Gelingen } \\
\text { der Täterstrategien schienen gegeben: } \\
\text { Aufmerksame Zuwendung des Täters } \\
\text { gegenüber den Jungen, ,interessante } \\
\text { Attribute des Täters (technisches } \\
\text { Verständnis und eine offenbar ausgeprägte } \\
\text { Bereitschaft, den Jungen ,Spannendes zu } \\
\text { zeigen“) und eine gemeinsame } \\
\text { Interpretation von Männlichkeit, die darin } \\
\text { bestand, sich für Technik zu interessieren } \\
\text { [...].“(Falldokumentation 3.1) }\end{array}$ & $\begin{array}{l}\text { „In der Beratung thematisiert Constantin } \\
\text { sein Verhältnis zu P. und wie sich ihre } \\
\text { Unternehmungen - echte „Männersachen“ } \\
\text { - meistens um Technik drehten. P. habe eine } \\
\text { Drohne mit einer hochauflösenden Kamera } \\
\text { besessen. Mehrmals haben sie sich mit dem } \\
\text { Tor-Netzwerk verbunden. Constantin wirkt } \\
\text { auf den Berater fasziniert und stolz.“ } \\
\text { (Fallvignette Constantin, s. Kap. 6) }\end{array}$ \\
\hline $\begin{array}{l}\text { „Die Eltern haben das Smartphone an sich } \\
\text { genommen und die monatelangen } \\
\text { Chatverläufe gelesen und dokumentiert. } \\
\text { Diese Chats wurden vom Täter hochgradig } \\
\text { pornografisch gestaltet.“ } \\
\text { (Falldokumentation 3.2) }\end{array}$ & $\begin{array}{l}\text { „Auffällig ist ein Chat, in dem [unser Sohn] } \\
\text { eindeutig pornografisch angemacht wird } \\
\text { [...]. Wir haben dann zu verstehen gegeben, } \\
\text { dass wir vom Chat wissen und darum } \\
\text { gebeten, uns sein Smartphone freizugeben.“ } \\
\text { (Fallvignette Eli, s. Kap. 5) }\end{array}$ \\
\hline
\end{tabular}

den Dialog gegangen. In jeder Focus Group wurde eine Fallvignette Schlüsselsituation um Schlüsselsituation diskutiert. Es ging dabei hauptsächlich um die Frage, welche Intervention(en) auf welcher Bemessungsgrundlage und unter welchen Gegebenheiten folgen sollen. Die persönliche fachliche Positionierung geriet durch das Aufeinandertreffen unterschiedlicher Perspektiven in Begründungsund Rechtfertigungszwang. Dies gewährte dem Projektteam Einblicke in die dahinterstehenden Überzeugungen, (Vor-)Annahmen und Grundsätze.

Perspektiverweiternd wurde das Erfahrungswissen von Menschen, die mediatisierte sexualisierte Gewalt in Kindheit und Jugend (mit-)erlebt haben, mittels leitfadengestützter Interviews erhoben. Die 19 Interviews $\left(\mathrm{n}_{\text {Gewaltbetroffene }}=12\right.$, $\mathrm{n}_{\text {Eltern von Gewaltbetroffenen }}=4, \mathrm{n}_{\text {Sozialarbeiter*innen }}=3$ ) wurden vorwiegend in spezialisierten Fachstellen durch geschultes Personal durchgeführt, um bei Bedarf eine adäquate Weiterberatung und/oder Krisenintervention zu ermöglichen. Der Fokus lag auf Anliegen und Ressourcen, die sich aus der Betroffenenperspektive in der Phase der Aufdeckung und während der professionellen Unterstützung und Begleitung ergeben.

Auf diese Weise sammelten wir auf ca. 1200 DIN-A4-Seiten transkribierten Datenmaterials die Erfahrungsexpertise von Gewaltbetroffenen und Expert*innen aus Wissenschaft und Praxis. Daraus leiteten wir die fachlichen Leitplanken in 
der Arbeit zu mediatisierter sexualisierter Gewalt ab. Zugleich arbeiteten wir die Grundpfeiler der Handlungsempfehlungen heraus. Hierfür griffen wir auf die sogenannte Reflexive Grounded Theory (Breuer et al., 2019) zurück. Durch das Starkmachen des reflexiven Moments flossen die Perspektivität und Subjektivität im Kontext von Hilfen in die Entwicklung der Handlungsempfehlungen ein.

„Ich war bei einer Ärztin. Weil ich sie fragen wollte, ob ich mir einfach etwas bescheinigen lassen kann, womit ich dann meine Ausfälle in der Schule begründen könnte. [...] Und dann hat sie gesagt, ja, ,du hättest ja einfach Skype ausmachen können. Du hättest ja einfach diese Person blocken können. Das ist ja nicht mein Problem`. Und das zu einer Person zu sagen, die gerade aus einem sexuellen Missbrauch rauskommt."

Gewaltbetroffene, Interview

Das im HUMAN-Projekt interviewte Mädchen schildert in obigem Zitat ihre Erfahrungen recht unmittelbar nach der ihr widerfahrenen Gewalt. Das Mädchen kann einerseits klar benennen, was sie damals gebraucht hätte, eine Krankmeldung und Verständnis statt Schuldzuschreibung zum Beispiel. Unbeantwortet ist damit aber noch immer die Frage, wie gesamtgesellschaftlich ein Bewusstsein dafür geschaffen werden kann, dass auch bei online verübter Gewalt Betroffene keine Schuld tragen. Das beispielhafte Zitat veranschaulicht zweierlei. Erstens wird die Bedeutsamkeit von Perspektivität deutlich. Während die Ärztin nicht zuletzt durch das Zuweisen einer Mitschuld die Gewaltwiderfahrnisse des Mädchens bagatellisiert, nehmen wir an, dass die Lesenden unserer Einordnung folgen, wonach Betroffene mediatisierter sexualisierter Gewalt keine Mitschuld tragen. Nichtsdestotrotz braucht es in der Arbeit mit dem Mädchen einen Umgang mit derlei Bagatellisierungen. Zweitens enthält das Datenmaterial einerseits konkrete Hinweise darauf, wie Konflikte im fachlichen Umgang mit dem konkreten Fall gelöst werden können. Andererseits sind einige Lösungsansätze abstrakt, fallabhängig, wurden von den Expert*innen kontrovers diskutiert oder aber führten zu weiteren offenen Fragen, die unbeantwortet blieben. Daher sei abschließend festzuhalten, dass wir den von den Expert*innen mit den Diskussionen erarbeiteten Handlungsrahmen durch eine wissenschaftlich fundierte Auseinandersetzung mit interdisziplinären Theorien, Diskursen, Schulen, pädagogischen und therapeutischen Ansätzen sowie rechtlichen Rahmenbedingungen in Handlungsempfehlungen übersetzt haben. 


\section{Literatur}

Bahls, C., Eßer, F., Hölling, I., Hüdepohl, G., Müller, S., Pluto, L., Rusack, T., \& Schlingmann, T. (2018). Partizipative Forschung - Memorandum. In A. Retkowski, A. Treibel, \& E. Tuider (Hrsg.), Handbuch Sexualisierte Gewalt und pädagogische Kontexte: Theorie, Forschung, Praxis (S. 1006-1008). Beltz Juventa.

Bergold, J., \& Thomas, S. (2012). Participatory research methods: A methodological approach in motion. Forum: Qualitative Sozialforschung, 13(1, Art. 30).

Bohnsack, R., \& Przyborski, A. (2009). Gruppendiskussionsverfahren und Focus Groups. In R. Buber \& H. H. Holzmüller (Hrsg.), Qualitative Marktforschung (S. 491-506). Gabler.

Breuer, F., Muckel, P., \& Dieris, B. (Hrsg.). (2019). Reflexive Grounded Theory: Eine Einführung für die Forschungspraxis (4. Aufl.). Springer VS.

forschungsdaten.info. (2019). Datenschutzrecht. https://www.forschungsdaten.info/themen/ rechte-und-pflichten/datenschutzrecht/. Zugegriffen: 5. Jan. 2021.

Kärgel, K., \& Vobbe, F. (2020). Mediatisierte Gewalt - Diffusion - Transzendenz: Erscheinungsformen und Herausforderungen sexualisierter Gewalt mit digitalem Medieneinsatz. Kindesmisshandlung und -vernachlässigung, 23(1), 30-43.

Kelle, U., \& Kluge, S. (Hrsg.). (2010). Vom Einzelfall zum Typus: Fallvergleich und Fallkontrastierung in der qualitativen Sozialforschung (2. Aufl.). Springer VS.

Kunz, R., Merten, G., \& Roller, C. (2016). Schlüsselsituationen der Sozialen Arbeit - ein Reflexions- und Diskursmodell. Sozialmagazin, 9-10, 67-73.

Tov, E., Kunz, R., \& Stämpfli, A. (2016). Schlüsselsituationen der Sozialen Arbeit: Professionalität durch Wissen, Reflexion und Diskurs in Communities of Practice. hep Verlag.

Open Access Dieses Kapitel wird unter der Creative Commons Namensnennung 4.0 International Lizenz (http://creativecommons.org/licenses/by/4.0/deed.de) veröffentlicht, welche die Nutzung, Vervielfältigung, Bearbeitung, Verbreitung und Wiedergabe in jeglichem Medium und Format erlaubt, sofern Sie den/die ursprünglichen Autor(en) und die Quelle ordnungsgemäß nennen, einen Link zur Creative Commons Lizenz beifügen und angeben, ob Änderungen vorgenommen wurden.

Die in diesem Kapitel enthaltenen Bilder und sonstiges Drittmaterial unterliegen ebenfalls der genannten Creative Commons Lizenz, sofern sich aus der Abbildungslegende nichts anderes ergibt. Sofern das betreffende Material nicht unter der genannten Creative Commons Lizenz steht und die betreffende Handlung nicht nach gesetzlichen Vorschriften erlaubt ist, ist für die oben aufgeführten Weiterverwendungen des Materials die Einwilligung des jeweiligen Rechteinhabers einzuholen.

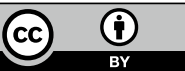

